\title{
Geometric Models of Atomic Nuclei
}

\author{
Gábor Bihari \\ Department of Experimental Physics, University of Debrecen, Debrecen, Hungary \\ Email: bihari.gabor@science.unideb.hu
}

How to cite this paper: Bihari, G. (2017) Geometric Models of Atomic Nuclei. World Journal of Nuclear Science and Technology, 7, 206-222.

https://doi.org/10.4236/wjnst.2017.73017

Received: June 6, 2017

Accepted: July 18, 2017

Published: July 21, 2017

Copyright (C) 2017 by author and Scientific Research Publishing Inc. This work is licensed under the Creative Commons Attribution International License (CC BY 4.0).

http://creativecommons.org/licenses/by/4.0/ c) (i) Open Access

\begin{abstract}
While experimenting with the more and more popular neodymium magnetic ball sets, the author developed a method, by which models of atomic nuclei can be created. These macroscopic models visually represent several features of nuclei and nuclear phenomena, which can be a useful mean during the teaching of nuclear physics. Even though such macroscopic models are unable to depict the true quantum physical nature of nuclear processes, they can be much more useful didactically than the previously used disordered sets of balls, to represent the atomic nucleus.
\end{abstract}

\section{Keywords}

Nuclear Model, Magnetic Ball, Rotational Symmetry, 4-Fold Symmetry, Magic Number, Shell-Orbit Structure, Deformed State, Cluster Model, Collective Excitation

\section{Introduction}

While teaching nuclear physics, a fundamental need can be experienced quite often to illustrate somehow the basic properties of atomic nuclei. Currently the representations of atomic nuclei are usually disordered sets of two differently colored ball-like objects (see Figure 1). However, this depiction is quite rudimentary, as none of the most important properties of atomic nuclei are appearing on these models.

Obviously a three-dimensional model can help more in the visualization of a nucleus, than a two-dimensional representation. The nowadays more and more popular neodymium magnetic ball sets can be a very good tool for the threedimensional modeling of the nucleus. Initially the author used disordered spherical sets of two differently colored magnetic balls to illustrate the nuclei. But after becoming more familiar with the possibilities these magnetic ball sets can provide, the author realized that some symmetric forms are more practical, as they can represent certain properties of the atomic nuclei: the effect of the 


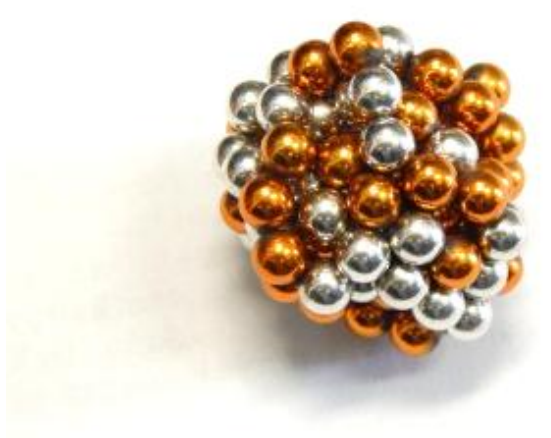

Figure 1. An atomic nucleus is usually depicted as a disordered set of particles.

Pauli principle, symmetries, deformed states, magnetic moments and even excitations. Thus, we are going to see in the following sections, that these magnetic ball sets offer surprising possibilities for the modeling of atomic nuclei.

\section{Why Macroscopic Objects-Sets of Magnetic Balls-Can Be Used to Model the Nucleus?}

Although it seems a bit primitive method to model the nucleus by setting macroscopic spheres into symmetric forms, due to some reasons, this idea is not entirely a hopeless attempt. For these reasons, the creation of geometric models can prove to be a very eloquent mean of nuclear physics education.

Some of these reasons are the consequences of the properties of nucleons. The nucleons-protons and neutrons are complex objects, made of other particles. This means that they can never be point-like objects at any energy: their minimum size is $0.8 \times 10^{-15} \mathrm{~m}$. To know the actual expanse of the wave function of the nucleons, one must know their mean energy in the nucleus. We know that from theory and experiment as well: it is about $20-30 \mathrm{MeV} /$ nucleon. At this energy the De Broglie wavelength of the nucleons appears to be roughly the same as their actual size: $0.5-0.8 \times 10^{-15}$ meters. That is, the probability amplitude of position does not exceed significantly the physical boundaries of the nucleon, as a complex system. To associate a graphic image to the nucleons of an atomic nucleus: they are more like spheres that ripples only at the edges-just as a blurry image of a defocused camera-than actual waves.

Thus, despite the small size of the nucleons and their quantum physical nature, due to their relatively large mass and energy, a classical description is not necessarily false. Fortunately, the velocity of the nucleons in the nucleus is moderate, so we do not have to deal with relativistic effects as well. This means that the average $20-30 \mathrm{MeV}$ energy of the nucleons is a kind of a window for classical approximations: at lower energies one cannot take a step without the use of quantum mechanics, while at higher energies relativistic effects are distorting the picture. However this energy gap, the mean energy of the nucleons in an atomic 
nucleus allows the possibility for a classical approach: at these energies the classical models are not completely useless.

It is a fortunate coincidence that the magnetic force generated between the dipole moments of the balls of the models, has similarities to the strong force between the nucleons for an extent. Namely, the strength of both forces decrease very rapidly with increasing distance, so they both have an effect only on neighboring objects. Again it is true for both interactions that the lines of forces prefer to close in the shortest possible distance. These features allow us for an extent to use the magnetic force as the analogy of the strong force between the nucleons.

\section{Symmetric Shapes and the Pauli Principle}

The packing of same sized spheres is a several hundred years old problem in mathematics. One of the first and most important statements of this topic is, that the packing density of ordered structures is usually much higher, than that of the disordered forms. Disordered forms fill maximum $64 \%$ of the available spaceleaving 36\% useless-while ordered symmetrical shapes can reach up to $74 \%$ packing density [1]. Due to the binding forces, the nucleons are also pursuing to minimize the space they occupy, when forming the nucleus. Thus, even though there are other factors that must be taken into consideration, the maximal packing density principle suggest that one must examine the role of the ordered, symmetrical structures, when modeling the nucleus.

There are two kinds of ordered structures, into which same sized spheres can organize: lattices and rotationally symmetric structures. Both types are characterized by the symmetries of the sets. The well-known lattices have translational symmetries in several directions. The other kind of symmetry is the rotational or radial symmetry: there are structures that can be rotated with a certain degree, and the rotated object will be identical to the original one (see Figure 2). The angle of rotation must be a definite portion of $360^{\circ}$ : if the degree in question is $360^{\circ} / \mathrm{n}$, we speak of $\mathrm{n}$-fold symmetry. For example the threefold symmetry of a

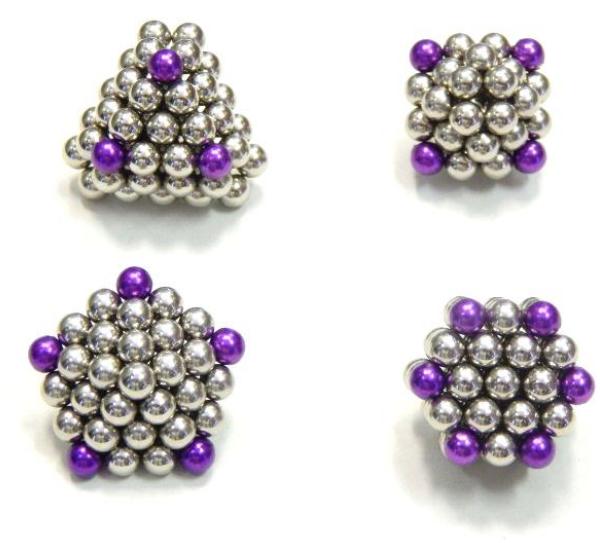

Figure 2. Structures of 3, 4, 5 and 6-fold rotational symmetry. With the proper rotation, the colored balls are replacing each other. 
structure means, that a rotation leaves the object unchanged, if the angle of rotation is $360^{\circ} / 3=120^{\circ}$ or its multiples.

A basic feature of lattice structures is that the number of particles in the lattice is not limited by any restriction. By the characteristic translations of the grid, in principle one can increase the number of particles in the lattice to infinity, nevertheless it remains the same lattice. In the case of the structures with rotational symmetry there are some restrictions however. The number of particles is unlimited in this case as well, but the rotational symmetry can appear only at certain particle numbers. If we add a new sphere to a structure of n-fold symmetry, the symmetry breaks, until further spheres are added to the structure. In the case of three-fold symmetry, three spheres are needed to complete the new structure. In the case of four-fold symmetry, four spheres are necessary, and so on: in the case of $n$-fold symmetry, $n$ spheres are necessary to create a new structure, which again have $n$-fold symmetry. Of course, the new spheres must be placed in such a way, that they replace each other by the characteristic rotation of the symmetry.

The two groups, the lattices and the structures with n-fold symmetry are interlinked in some cases. Some structures have not only rotational, but translational symmetries as well. These structures are indeed such kinds of lattices, which have rotational symmetry. Let's think for example of a two-dimensional honeycomb structure: it can have three and six-fold rotational symmetry, but it is created as a lattice, by placing particles at given distances. There are exceptions however: no lattice exists with five-fold symmetry for same sized spheres [2].

The detailed examination of various lattices and rotationally symmetric structures is beyond the scope of this article. Fortunately, we do not need such details at all. Even the study of the simplest forms can lead to the conclusion that for modeling atomic nuclei, only structures of 4-fold symmetry need to be considered. When creating spherical structures with 3-, 4-, 5- or 6-fold symmetries, one does not observe any special feature regarding the objects with 3-, 5- or 6-fold symmetries. However, the numbers of particles in the 4-fold structures are reminiscent to magic numbers of nuclear physics. Those compact structures are more or less spherical, and have 4-fold rotational symmetry, number 4, 16 and 40 particles. This is identical to the numbers of nucleons in the most stable nuclei-the ${ }^{4} \mathrm{He},{ }^{16} \mathrm{O}$ and ${ }^{40} \mathrm{Ca}$ nuclei-in which both the protons and the neutrons have so called magic numbers. In nuclear physics, these numbers are indicating closed shell structures.

In the case of 3, 5 or 6-fold symmetry, there is no such coincidence. One can create shell-like structures with 3,5 or 6-fold symmetries, and by uniting them, it is possible to create compact, spherical objects. But in these cases, the numbers of balls in the structures are definitely unlike any special number appearing in nuclear physics. Indeed, in the case of 5-fold symmetry, very compact, durable, almost "solid" structures can be created. But the numbers of particles in these structures have no connection with nuclear physics. For example, the diamond-shaped pentagonal bipyramids number $7,23,54,105,181$, etc. particles 
(see Figure 3). These numbers have no special meaning in nuclear physics. In the case of 3- or 6-fold symmetries, it is very difficult to create spherical objects, as these numbers are usually creating planar structures: 2 or 3 dimensional lattices. Nevertheless, the task is not impossible, but the created structures again show worthless particle numbers. So these symmetries-3, 5 or 6-fold rotational symmetries-are alluring with less success even at the beginning.

The similarity between the magic numbers of nuclear physics and the number of particles in the world of the objects with 4-fold symmetry can help us to follow an easy way to create useful models of atomic nuclei. The case is indeed quite simple, and after understanding the cause of this similarity, it seems to be an obvious possibility to model the nucleus with symmetrical objects.

According to the Pauli principle, the nucleons of the atomic nuclei-as any other fermionic particle-can occupy the possible states in limited numbers. In the case of nucleons the highest possible number is four: two protons and two neutrons can occupy each state with opposite spins. Thus, any visualization of the Pauli principle has to use such structures, which have 4-fold symmetry: in this way every state allows four identical positions to place a particle. Not more, not less, but exactly four particle should sit in equivalent spatial positions in the models. And this requirement can be fulfilled only with structures of 4-fold symmetry.

In this way, we found our first principle, which help us to create geometric models of atomic nuclei: it is indeed the geometric visualization of the Pauli principle. The massiveness, the denseness of the nucleus can be interpreted by a simple disordered set of magnetic balls. But, if we would like to visualize other important nuclear features, like Pauli's principle, than we have to use symmetric structures-in this case those with 4-fold rotational symmetry. In these macroscopic models every "state" is filled with four magnetic balls, placed in four equivalent spatial positions. Just like in the nucleus, where every state is filled with four nucleons.

\section{Structures with 4-Fold Symmetry and the Nuclear Shell Model}

The structures, that have 4-fold rotational symmetry-just as any other symme-
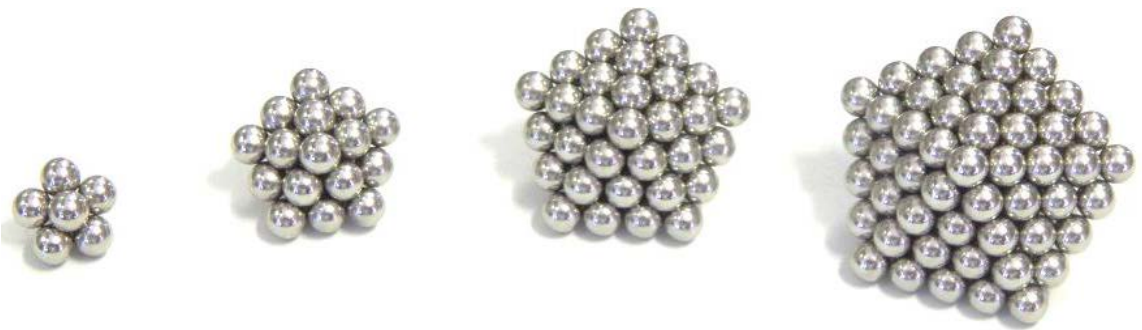

Figure 3. Pentagonal bipyramids with increasing particle numbers. The complete structures number 7, 23, 54, 105 particles. The particle numbers of such structures have nothing in common with nuclear structures, except for the case of some structures of 4 -fold symmetry. 
trical structures-can be created from smaller symmetrical units. In the case of lattices, these smaller units are the unit cells, which are repeated along the axes of symmetry. Whereas in the case of structures with rotational symmetry, these units are rings, which create planes or curved shells, that can be organized further into complex objects. In the case of 3 or 6-fold symmetry, the balls are organized into flat planes, without any curvature, while in the case of four or fivefold symmetries, tightly packed balls cannot form flat surfaces. In these cases shell-like structures are formed with a convex and a concave side, and thus, these three dimensional shells can obviously be organized into three dimensional objects.

In the case of 4 -fold symmetry, the building blocks, the rings should be made of $4,8,12$ etc. balls. The numbers of balls in the rings are always multiples of four: $4 \mathrm{n}$. One can build a shell-structure of these rings starting with the smallest one and continuing with rings of increasing size. The simplest shell is made of only one ring, the smallest ring with 4 balls. The next shell consists of two rings that are made of 4 and 8 balls. The third shell consists of three rings, made of 4,8 and 12 particles, and so on (see Figure 4). As these numbers are forming an arithmetic series, we know that the number of balls in the shells are $N=2 n(n+1)$ : $4,12,24$, where $\mathrm{N}$ is the number of balls in the $\mathrm{n}^{\text {th }}$ shell.

Of these hemi-spherical shells one can create spherical objects. These structures are made of the first, the first two, the first three, etc. shells consisting $4,4+(4+8)=16$ and $4+(4+8)+(4+8+12)=40$ particles. To continue the building after the spherical structure of 40 balls, we have to fit the fourth domed shell, which is a bit difficult, but possible task. This structure will count $4+(4+8)+(4+8+12)$ $+(4+8+12+16)=80$ balls (see Figure 4$)$.

We could proceed further ahead, but the main point can be seen at this stage

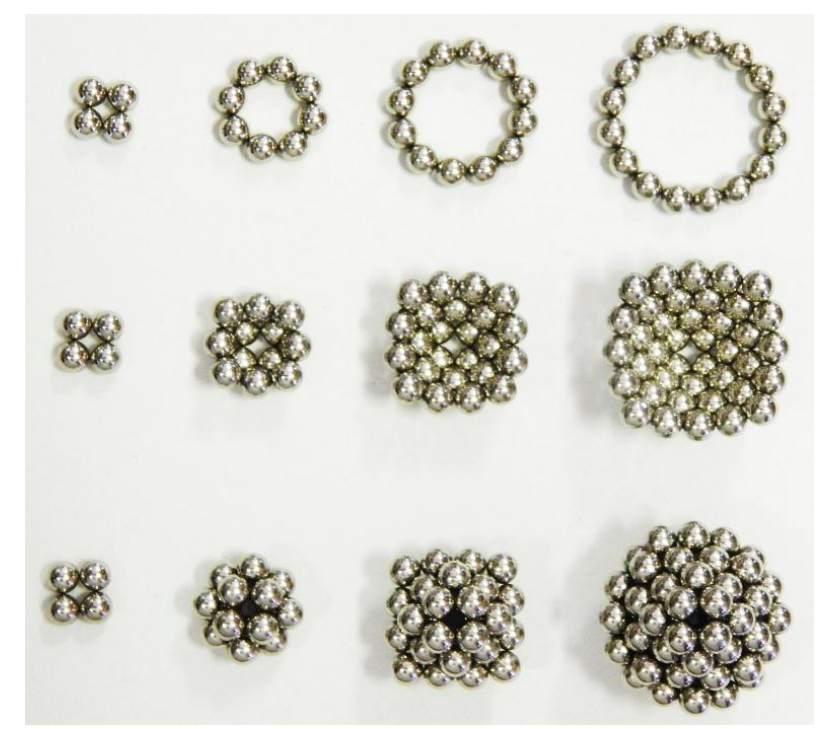

Figure 4. Rings, shells and spheres with 4 -fold symmetry. The spherical objects number 4,16 and 40 particles, just as the most stable nuclei. The cause of similarity is simply the visualization of Pauli's principle. 
already. In the first three cases there is a perfect match with the particle numbers of the most stable atomic nuclei. The first model can be associated with the ${ }^{4} \mathrm{He}$ nucleus. The second model corresponds to the ${ }^{16} \mathrm{O}$ nucleus, while the third one to the ${ }^{40} \mathrm{Ca}$ nucleus. Nevertheless in the fourth case, the particle number of the fourth model does not meet with that of any outstandingly stable nucleus. Indeed, the first attempts of the shell model of atomic nuclei proposed an island of extreme stability to the particle number 80 . But reality tells a different story: due to the increasing repulsion of positively charged protons, beyond ${ }^{40} \mathrm{Ca}$, the nuclei with identical proton and neutron numbers are no longer stable. On the other hand, the interaction of the angular momenta of particles-the spin and orbital momentum-further complicates the situation, so the next particularly stable nuclei have 50 protons or neutrons.

Thus, modeling the nuclei above atomic number 40 is a difficult task. Nevertheless, for the light nuclei there is a chance to demonstrate the shell model of the nucleus in a simple form. The observation that the light nuclei prefers the same, magic" numbers as the objects of four-fold rotational symmetry, highlights the possibility for visualizing the shell model of nuclei on our models. Such a demonstration of the nuclear shell model can be a very useful aid in education, as it attaches a macroscopic picture to an abstract physical phenomenon. Although, as teachers we would like to avoid superficial approaches, such a palpable model can simplify the process of understanding.

According to the nuclear shell model, the structures of the above nuclei are: ${ }^{4} \mathrm{He}: 1 \mathrm{~s}^{1 / 2} ;{ }^{16} \mathrm{O}: 1 \mathrm{~s}^{1 / 2}, 1 \mathrm{p}^{3 / 2}, 1 \mathrm{p}^{1 / 2} ;{ }^{40} \mathrm{Ca}: 1 \mathrm{~s}^{1 / 2}, 1 \mathrm{p}^{3 / 2}, 1 \mathrm{p}^{1 / 2}, 1 \mathrm{~d}^{5 / 2}, 2 \mathrm{~s}^{1 / 2}, 1 \mathrm{~d}^{3 / 2}$-for protons and neutrons as well. This means that the energy structure of the above nuclei consist energy levels on which two protons and two neutrons, or four protons and four neutrons, or six protons and six neutrons can be found. In total, these are 4, 8 or 12 particles. Our previous magnetic models are made of rings that consist of 4,8 or 12 magnetic balls. Thus the analogy is promising. If we would like to demonstrate the energy and shell structure of the atomic nuclei, we have to see the rings of the macroscopic models as kind of "orbits". These rings can symbolize the energy levels or "orbits" of the nucleus. At the same time, the shells of the models, made of rings can symbolize the shells of the nucleus, made of several different orbits.

The first nuclear shell, the 1s shell is made of one orbit, one energy level that holds four nucleons: it is symbolized with the 4 ball ring. The second $1 p$ shell is made of two energy levels, the $1 p^{3 / 2}, 1 p^{1 / 2}$ levels, which accommodate 8 and 4 nucleons respectively. The analogy of this nuclear shell in the macroscopic model is the shell that is made of a 4 and an 8 ball ring. The third shell that is formed in the nuclear potential well is built of three energy levels: the $1 \mathrm{~d}^{5 / 2}$ and the $1 \mathrm{~d}^{3 / 2}$ levels, and the $2 \mathrm{~s}^{1 / 2}$ level. They are accommodating 12,8 and 4 nucleons, and thus, it is obvious that the third shell of our macroscopic model-with 4, 8 and 12 ball rings - can represent this third nuclear shell.

Along with the shells of the nuclear shell model, we can obtain an expressive image on one more feature of the energy levels, the "orbits". As it was mentioned 
before, regarding the Pauli principle, only 4 particles can occupy the same quantum state in a nucleus. Nevertheless, we have energy states that are filled with 8, 12 etc. nucleons, which means that these energy levels are degenerate: they are made of several different states with identical energy. This feature of the nucleus can be easily demonstrated on the ${ }^{16} \mathrm{O}$ model for example. In this model the 8 ball ring goes along a zigzag line, while encircling the model. In other words, four of the 8 balls are in higher positions than the other four balls. They are part of the same ring, but there are two different spatial positions available for the balls in this ring. And only four are occupying each of these positions (see Figure 5). However, these spatial states are equivalent each other: this becomes obvious as the model is turned upside down and the states change their positions-while the model is the same and undistinguishable from the original form.

The models of the nuclear orbits, that is, the rings of the geometric models help to visualize one more interesting feature of the atomic nuclei: the angular momenta of the nucleons. The analogy is not perfect, though: the angular momentum is a feature that is impossible to display on macroscopic level. But, at least there are some similarities between the models and the quantum mechanical reality. Namely the models can show the difference of angular momentum on the $s, p, d, f$ etc. orbits. It is easy to see, that the more balls a ring contain, the larger is its diameter. And so, if we rotate the models-either in reality or just in imagination-the larger rings will have larger angular momentum than the smaller ones. The 4 ball ring will have the smallest angular momentum, the 8 ball ring will have larger. The 12 ball ring outside of them will have even larger angular momentum-although all the rings are rotating together in one single model.

\section{Other Models with Four-Fold Symmetry}

The above thoughts are mostly based on the characteristics of the ${ }^{16} \mathrm{O}$ and ${ }^{40} \mathrm{Ca}$ models. Once, however, we managed to find parallels between the nuclear shell

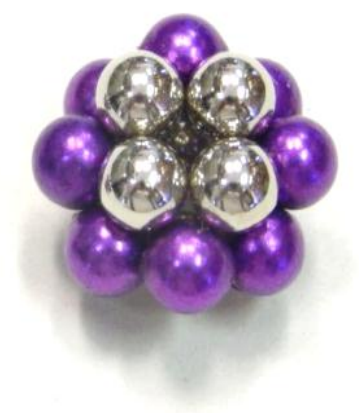

Figure 5. The degenerate $1 \mathrm{p}^{3 / 2}$ orbit of the ${ }^{16} \mathrm{O}$ nucleus is shown on this magnetic model with colored balls. The four balls that form a + shape is one state of the orbit, while the other four colored balls, creating an $\mathrm{X}$ shape is the other state of the orbit. 
model and the geometric models, we have the guidance to build the geometric model of any nucleus. All we need to know for modeling is the shell structure of the nucleus in question: which states are occupied by the nucleons. We construct the appropriate rings, depicting the completed states and orbits and then interconnect them for creating the model, which illustrates several features of the nucleus in question.

A simple nucleus for example is the ${ }^{12} \mathrm{C}$. According to the shell model, there are three orbits in this nucleus: $1 \mathrm{~s}^{1 / 2}, 1 \mathrm{p}^{1 / 2}, 1 \mathrm{p}^{3 / 2}$. The first two accommodate only four nucleons, and even though the third one could be filled with 8 nucleons, there are only four on it-two protons and two neutrons. So, every orbit of this nucleus contains four particles. The model of the ${ }^{12} \mathrm{C}$ thus comprises two 4-ball rings and four separate balls that actually do not form a visible ring (see Figure 6). The easiest way to build the model is to create the second shell of 12 balls: a 4 and an 8 ball ring, joined together. Then, the only thing we need is to push the four corner of the larger ring to join them together-thus four balls move from the $1 \mathrm{p}^{3 / 2}$ orbit to the $1 \mathrm{p}^{1 / 2}$ orbit.

Another interesting example is the geometric model of the ${ }^{20} \mathrm{Ne}$ nucleus. The shell structure of this nucleus is similar to the ${ }^{12} \mathrm{C}$, as both nuclei have half-vacant shells. The ${ }^{20} \mathrm{Ne}$ nucleus has three completely filled orbits: a $1 \mathrm{~s}^{1 / 2}$ and a $1 \mathrm{p}^{1 / 2}$ orbit with 4-4 nucleons, and a $1 \mathrm{p}^{3 / 2}$ orbit with 8 nucleons-these are indeed the complete orbits of the ${ }^{16} \mathrm{O}$. While there is an orbit with only 4 nucleons: the $1 \mathrm{~d}^{3 / 2}$ orbit. The easiest way to create the geometric model of such a nucleus is to use three rings with $4-4$ and 8 balls and 4 separate balls. We have to fit one of the 4 ball ring to the 8 ball ring. Than the four separate balls have to be fitted to the open side of the 8 ball ring, which create a zig-zag line out of the 8 ball ring. This line can be closed with the only left 4 ball ring.

An interesting feature of this model is that, it is impossible to distinguish the $1 \mathrm{p}^{3 / 2}$ and $1 \mathrm{~d}^{3 / 2}$ orbits from each other. They create a ring that contains 1 ball2balls-1ball-2balls etc. in a row (see Figure 6). Even though this feature has only a superficial similarity to the pairing of quantum mechanical states, one can use it to demonstrate the latter.

The next model after the ${ }^{20} \mathrm{Ne}$ with four-fold rotational symmetry is the model of ${ }^{24} \mathrm{Mg}$ that contains 4 more balls. The orbit structure of the ${ }^{24} \mathrm{Mg}$ nucleus is very simple. It comprises four completely filled orbits: there are 4 nucleons on the $1 \mathrm{~s}^{1 / 2}$ and the $1 \mathrm{p}^{1 / 2}$ orbits, and 8 nucleons on the $1 \mathrm{p}^{3 / 2}$ and $1 \mathrm{~d}^{3 / 2}$ orbits. That is, the

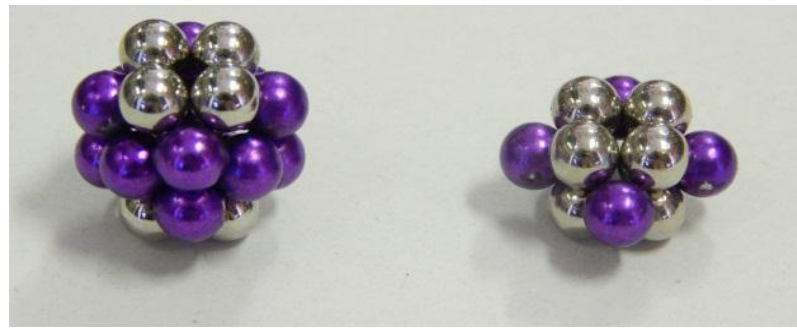

Figure 6. The geometric models of the ${ }^{20} \mathrm{Ne}$ and ${ }^{12} \mathrm{C}$ nuclei. The nucleons of the $1 \mathrm{p}^{3 / 2}$ and the $1 \mathrm{~d}^{3 / 2}$ orbits are represented with purple colored balls. 
model of this nucleus consists of two 4 and two 8 ball rings. It is very simple to build this model. We need to join the 4 and the 8 ball rings to form two hemispherical shells and then, these two hemispheres can be fitted together into a spherical model. The result is a surprisingly symmetrical model. It has not only one but three rotational symmetry axes, perpendicular to each other.

The ${ }^{24} \mathrm{Mg}$ model suggests two interesting ideas. One of them is related to the symmetry of the model. The observation of the three rotational symmetry axes of the model leads to the conclusion that the model can be interpreted as the group of 6 rings made of 4 balls (see Figure 7). But we have seen previously that the 4 ball ring-on its own-is the model of the ${ }^{4} \mathrm{He}$ nucleus itself. Thus, the model of the ${ }^{24} \mathrm{Mg}$ nucleus is indeed naturally made of $6{ }^{4} \mathrm{He}$ cluster. That is, unintentionally a cluster model of the ${ }^{24} \mathrm{Mg}$ nucleus is created.

Up till now, we did not dealt with the cluster model of the atomic nuclei, according to which the nuclei-and especially the deformed states of nuclei-can be interpreted as bounded states of several smaller, more stable nuclei. In the case of the ${ }^{24} \mathrm{Mg}$ model we found a natural overlapping of the shell model and the cluster model of the atomic nuclei. The geometric model of the ${ }^{24} \mathrm{Mg}$ nucleus was built by the help of the shell model, but the final picture can be interpreted with the help of the cluster model as well.

Another interesting feature of the ${ }^{24} \mathrm{Mg}$ model is the cavity inside the model. The inner cavity, which was created by the joining of two hemispheres, is not large enough to accommodate 4 balls. That is, the inner cavity must be unfilled. Due to the Pauli principle, our models have to have a four-fold rotational symmetry, and so, in the case of ${ }^{24} \mathrm{Mg}$, the inner cavity must be empty, if there is no space for 4 balls. No matter how logical it seems to fill the empty space inside the model, one can put only 1 - 2 or 3 balls inside. Nevertheless, this is unacceptable: for preserving the 4-fold symmetry, we must fit 4 balls to every possible state. Surprisingly, nuclear data can support such cavity in the inner core of light nuclei. It was observed in the case of several light nuclei, that the mass and charge density is decreased in the central part of the nucleus [3].

\section{Geometric Models of Deformed Nuclei and the Cluster Model}

Until now, it was a clear task to create the geometric models. Based on the shell-
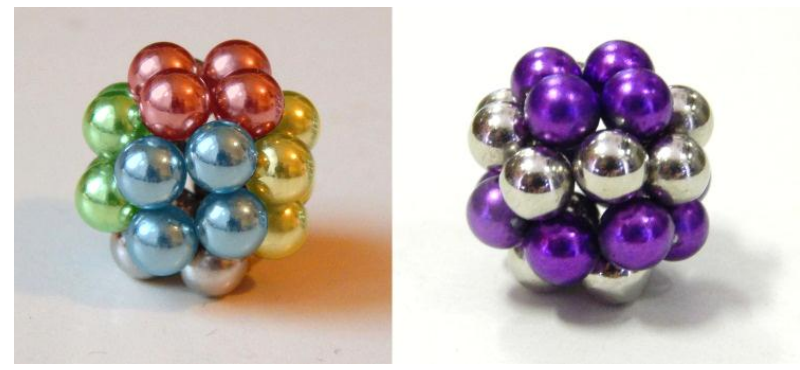

Figure 7. Two identical models of ${ }^{24} \mathrm{Mg}$. The first one show the ${ }^{4} \mathrm{He}$ clusters within the model with different colors, while the second one highlights the four orbits with different colors. 
orbit structure of the nuclei to be modeled, one can create the rings that correspond to the orbits of the nucleus and adjoining these rings finalizes the model. However, some special phenomena appear as we move away from the ${ }^{24} \mathrm{Mg}$ model with its particular symmetry and an inner cavity. When increasing the number of balls to 28 , the spherical symmetry breaks and the inner cavity is filled in several different ways. Thus, the next model with four fold symmetry, the ${ }^{28} \mathrm{Si}$ model can exist in several different forms, which is indeed very similar to the true nature of the ${ }^{28} \mathrm{Si}$ nucleus.

The ${ }^{28} \mathrm{Si}$ nucleus is a major milestone in the world of the nuclear structures. The smaller nuclei can have deformed states, but these states are forming only at very high excitation energies, and so, the deformed nucleus is at the verge of disintegration. Usually these deformed states are decaying with the emission of nucleons or ${ }^{4} \mathrm{He}$ nuclei, which indeed, means the disintegration of the nucleus. As a consequence of this, the deformed states of the light nuclei are not long lived metastable states, but in fact the first steps to their disintegration.

With the case of the ${ }^{28} \mathrm{Si}$ nucleus, we leave the realm of the light nuclei. The deformed states of the ${ }^{28} \mathrm{Si}$ nucleus are quite stable, so much so, that these metastable states have their own excited states, their own vibrational and rotational spectra. And beside the several long-lived deformed states, even the ground state of this nucleus itself is not spherical, but deformed. Thus, from the ${ }^{28} \mathrm{Si}$ nucleus, the world of medium heavy nuclei follows, where the nuclei often exist in several different shapes, with considerably long half-lives.

We know the various deformed states of the ${ }^{28} \mathrm{Si}$ nucleus quite well. The lowest energy state, the ground state is not a spherical form, but a slightly flattened shape, a so called oblate shape. The other, excited states may form strongly flattened oblate shapes, or elongated prolate shapes. Typically, the higher is the excitation energy of the state, the greater is the deformation [4].

Such deformed states can be illustrated easily with the geometric models made of magnetic balls. The 28 magnetic balls can form several different structures with four-fold rotational symmetry. None of them is spherical, just as in the case of the ${ }^{28} \mathrm{Si}$ nucleus. Some of them are shown in Figure 8, along with the orbit and shell structure of the nuclear states they correspond in Table 1.

The several deformed but rotationally symmetric models of the 28 balls can again contribute to the understanding of nuclear processes. Namely, the clustering of heavier nuclei in deformed states can be visualized by the help of these models. During nuclear experiments, it is often observed, that the elongated, prolate forms of heavy or medium nuclei can disintegrate in the presence of excess energy and thus creating two or three smaller nuclei. These decay products are usually the extremely stable ${ }^{4} \mathrm{He},{ }^{12} \mathrm{C},{ }^{16} \mathrm{O}$ nuclei. The accepted theory of this clusterization process is that inside the highly excited nucleus dense zones are formed and the later decay products are formed in these zones, before the actual decay. Thus, during the clustering process, the highly stable products are formed before the disintegration of the original nucleus.

The deformed models of the figure below can present a fitting example to this 


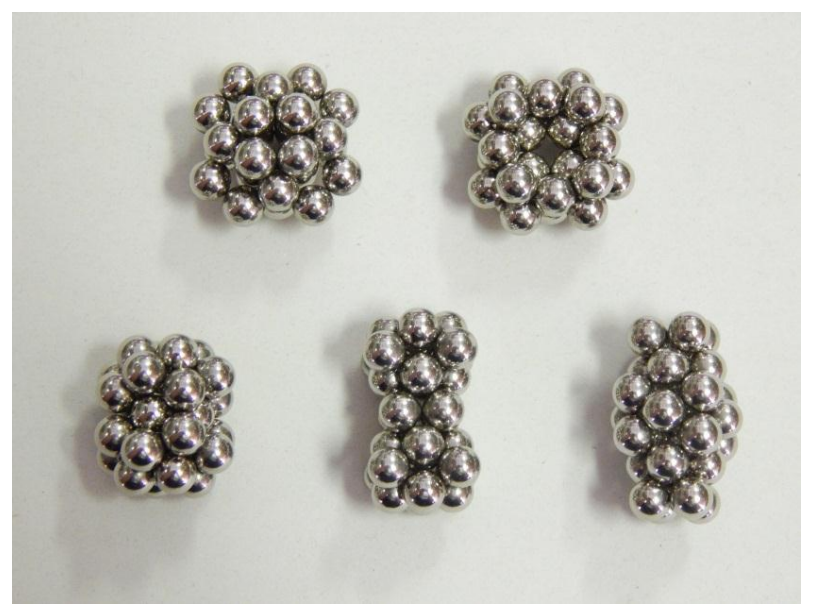

Figure 8. Structures of 28 magnetic balls with 4-fold symmetry. The axes of the upper, flattened shapes point outwards of the picture. The lower, elongated shapes situated so, that the axes are in the image plane, north-south direction. By these models, the deformed states of ${ }^{28} \mathrm{Si}$ nucleus can be visualized.

Table 1. Structures of the above macroscopic models in terms of the nuclear shell model. Beside the ground state (first model in the first line) the oblate excited state (second model in the first line) and the first prolate excited state (first model in the second line) is described.

\begin{tabular}{ccccccc}
\hline & $1 \mathrm{~s}^{1 / 2}(4)$ & $1 \mathrm{p}^{3 / 2}(8)$ & $1 \mathrm{p}^{1 / 2}(4)$ & $1 \mathrm{~d}^{5 / 2}(12)$ & $1 \mathrm{~d}^{3 / 2}(8)$ & $2 \mathrm{~s}^{1 / 2}(4)$ \\
\hline ground state & 4 & 4 & 4 & 8 & 4 & 4 \\
oblate excited & 4 & 8 & 0 & 8 & 8 & 0 \\
prolate excited & 4 & 8 & 4 & 0 & 8 & 4 \\
\hline
\end{tabular}

process. The elongated models can be considered as joined structures of smaller models, namely $\left({ }^{12} \mathrm{C}+{ }^{4} \mathrm{He}+{ }^{12} \mathrm{C}\right)$ or $\left({ }^{4} \mathrm{He}+{ }^{20} \mathrm{Ne}+{ }^{4} \mathrm{He}\right)$ models-see Figure 8 , second row. And in fact, these elongated structures can be actually prepared by the adjoining of these smaller models. That is, these deformed models can serve a very simple demonstration, as to how the clustering process appears in the highly excited and deformed states: indeed, they are made of smaller nuclei.

Along with the clustering process, the group of models on the above picture may illustrate us the phenomenon of collective excitations. In the case of collective excitations, the incoming energy is not concentrated on one single nucleon-these are the one-nucleon excitations-but several nucleons get simultaneously to a higher energy state. As the excitation energy changes the wave function of several nucleons-so to say, dislocates them" to another orbit-this kind of excitation often leads to a deformed state of the nucleus. Thus the collective excitation process and the deformed state of the nucleus are usually going along.

The exact explanation of these collective excitations is quite complex. A simplified view is that the deformed state, which is created by the excitation, with its deformed potential well shifts the energy levels of the nucleus. In this way, some of the excitation levels can be reached by the nucleons at much lower energies. 
So much so, sometimes 4 or 8 nucleons can be excited with smaller energy than one single nucleon. The excitation of one single nucleon usually does not affect the energy levels of a nucleus, but the excitation of several nucleons can do it. Thus, sometimes multi-nucleon transfer to another orbit can require less energy than a single-nucleon excitation.

The illustrative presentation of collective excitations is very simple by using the geometric models. The best way is perhaps to observe the transformation between the two oblate models of 28 balls. At the top of the model that corresponds to the ground state of the ${ }^{28} \mathrm{Si}$ nucleus, there is a 4 ball ring, which corresponds to an $s^{1 / 2}$ orbit. If the bounds of this ring is somehow breaks, the balls jump downwards to the $\mathrm{p}^{3 / 2}$ orbit below, which previously had 4 particles. By the dissolution of the upper $\mathrm{s}^{1 / 2}$ ring, the $\mathrm{p}^{3 / 2}$ ring under is now complete: it has 8 particles instead of 4 (see Figure 9).

Needless to say, that the dislocation of only 1 particle would need large amount of energy: the $s^{1 / 2}$ ring, a ${ }^{4} \mathrm{He}$ cluster must be broken, but at the same time the $\mathrm{p}^{3 / 2}$ ring is not filled with this single particle. When the $\mathrm{s}^{1 / 2}$ ring is completely dissolved and the $\mathrm{p}^{3 / 2}$ ring is thus filled, it needs much less energy than moving just one particle. Thus the energy balance between the breakup of bonds and creating new bonds can lead to the process of collective excitations and the change in the shape of the nucleus, the deformation.

It can be seen in this case, that a single particle excitation may consume large amount of energy, while the dissolution of an orbit and the completion of another creates a more balanced situation. Large amount of energy is needed to dissolve the bonds of an orbit, but almost the same energy can be released when another is completed with the same particles. And thus, a metastable state can form in the process with a new shell structure and shape. With the help of the geometric models, even the process can be visualized. By pushing the upper $s^{1 / 2}$ ring from upwards-with a pen for example-the ring breaks up and the balls

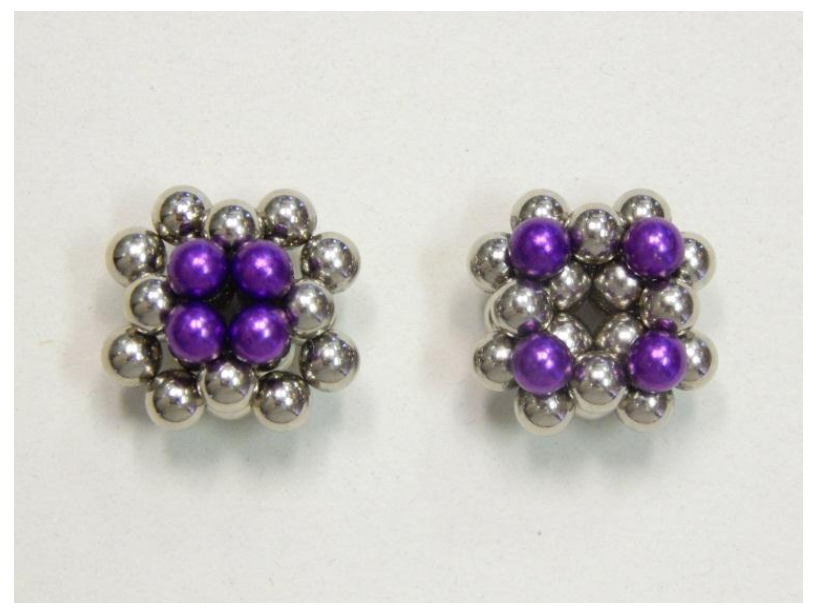

Figure 9. The two oblate models of ${ }^{28} \mathrm{Si}$ that helps to visualize the collective excitations. The purple colored four particle ring of the first model dissolves and the particles completing the 8 particle ring below. 
automatically jump to the hollows of the $\mathrm{p}^{3 / 2}$ ring, thus completing it. The process is reversible: by symmetrical lateral pressure, the 4 balls can be torn out of the $\mathrm{p}^{3 / 2}$ ring and by the attractive force they jump together again, forming the 4 ball $\mathrm{s}^{1 / 2}$ ring.

The preparation of elongated models is the simplest in the same way. For example, if one is pressing 4 balls of an 8 ball ring inwards, the balls are shifted to the inner parts of the model and two 4 ball ring are formed. In this way, the shape of the model can be changed, new orbits can be formed. Several such deformations can form the above mentioned clusters out of the original model. In the case of lighter nuclei, there are only 4 or 8 particles on the orbits, so the "collective excitations"-deformations of the models and the clustering process - can be illustrated in an easy and spectacular way.

\section{Magnetic Moment in the Geometric Models}

We have seen so far, that geometric models built of magnetic balls may help to illustrate several phenomena of the nuclear world for students. These models are not just disorderly packages of balls to represent the nucleons of an atomic nucleus. Symmetrical forms can illustrate the Pauli principle, the shell-orbit structure of a nucleus, the mechanism of collective excitations and deformation, and through these, the appearance of clustering process. These examples are more than enough to prove that such geometric models can be useful in illustrating the basic features of a nucleus while teaching nuclear physics. Nevertheless, there are even more possibilities.

One possibility is to take advantage of the magnetic nature of the balls, the models are made of. These balls have similar dipole magnetic field as the nucleons. For this reason, the magnetic moment of the balls gives us an opportunity to demonstrate the dipole moment of the nucleons. In those nuclei, which have even proton and neutron numbers, the spins and magnetic moments of protons and neutrons usually cancel each other. Thus, at least in the ground state, these nuclei usually have zero spin and magnetic moment.

The same is true for the geometric models, if they are made of magnetic balls. Looking at a ring that is part of a larger model, one may observe, that the magnetic field is basically goes around inside the circular rings. The dipole moment of a ball is a vector that point to one neighbor of the ball to the other: the vector is indeed parallel to the tangent of the ring at every ball. This means, that until a ring is complete, the magnetic moment of the ring is zero (see Figure 10). The magnetic moment of every ball is compensated by another ball, which is just in the opposite position of the ring. What is more, the balls which are opposite to each other, and thus compensating the magnetic moments of each other, are in equivalent geometric positions. A rotation by 180 degrees exchange these balls, which means that they are in the same "state": they are representing nucleons in the same state with opposite spins, regarding the Pauli principle.

One must not forget of course, that this macroscopic model is false by its birth, just as every other model. The nucleons have quite large energies in the 


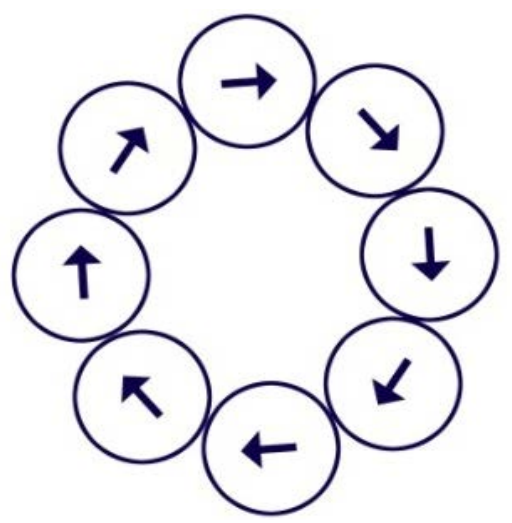

Figure 10. Direction of magnetic moments in a ring of magnetic balls.

nucleus, and thus, moving continuously in their common potential well. Their behavior can be described only by the help of quantum mechanics. Thus, it is meaningless to state that some nucleons of a nucleus are in opposite positions. But when visualizing the possibility that some nucleons are creating pairs and compensating the magnetic moments of each other, these models may give some help. So, even though the picture is false, it can help to visualize the intangible reality of the nucleus. When looking at the rings of the models, one can imagine how the angular moments and magnetic moments of the nucleons compensate each other. And also, how the spin and magnetic moment of the nucleus created by a single nucleon, if its pair is missing from the nucleus.

When visualizing the magnetic moments of the models, we do not need to rely only on our imagination. Although we cannot see the magnetic field of the nucleus, we can see that of the model. A very simple, age-old method gives us nice results: the magnetic field is made visible by laying a sheet of paper on the model and sprinkling fine iron powder on it (see Figure 11). The models have quite complex magnetic picture, which again gives food for some thoughts.

First of all, these magnetic images reveal, how important is the effect of closing the field lines. The model of ${ }^{4} \mathrm{He}$, a closed ring of four balls has a very weak field outside the model, compared to a linear arrangement of four balls. And even though we are seeing only the magnetic field of some macroscopic balls, one can imagine the similar effect of closure of field lines in the case of the strong nuclear force. And examining the magnetic properties of the more complex models, it becomes clear, that the models made of closed rings have very faint magnetic field outside the model. However, it is enough for one ball to be missing from the model; a burst of magnetic field appears on the picture (see Figure 12). Such pictures indeed, nicely illustrating, how the strong force burst out of those nuclei, which misses only one or two nucleons to have a closed shell. In the case of these nuclei the absorption cross section is much higher than in the case of nuclei with completely filled shells. And indeed, these models, that miss only one ball to fill a ring, drags a single magnetic ball from a much larger distance, than other models with closed rings. 


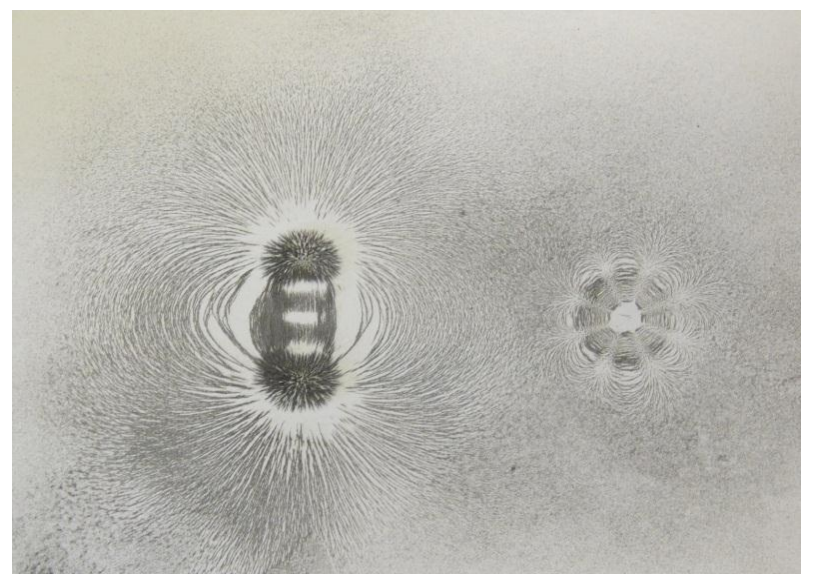

Figure 11. Magnetic field of the open line and closed ring of four magnetic balls.

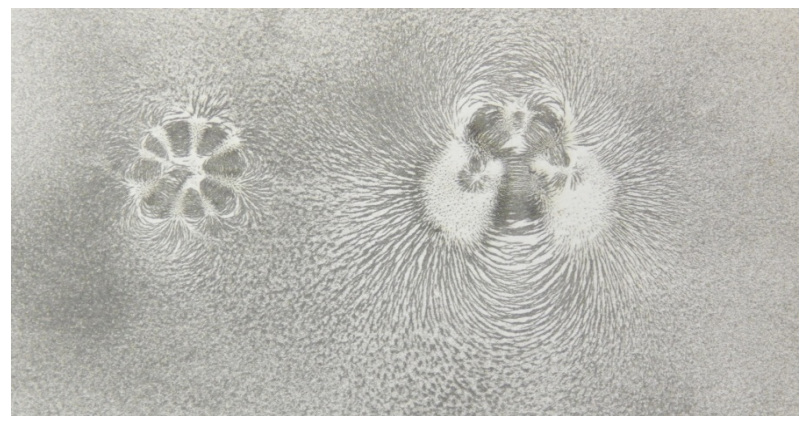

Figure 12. Magnetic field of the models made of 16 and 15 balls. The former model has only closed rings, while the latter has an open 7 ball ring. The burst of magnetic field highlights the position of the missing $16^{\text {th }}$ ball. Interestingly, the "missing ball field" is similar to the dipole field of a single ball.

\section{Concluding Remarks}

At first, it may seem to be a weird idea to model a complex, multi-particle quantum mechanical system by the help of macroscopic objects; we have seen that sometimes there are some similarities. The above thoughts have shown that modeling the atomic nucleus with sets of magnetic balls is not as weird idea as it may sound. Even though this modeling process has its limitations, these models might prove that the macroscopic visualization of nuclear features is not a completely hopeless task. These models may give a tangible picture as to how the piles of nucleons can arrange to such complex structures as the atomic nuclei.

Due to the spatial limitations of this article, several interesting topics had to be neglected. For example, 3-fold symmetry may apply for the modeling of those nuclei, in which there are two times more neutrons than protons or more protons than neutrons. In these cases there are only three nucleons in every state, so the models have to have 3-fold symmetry. Again, interesting structures appear, when the protons and neutrons are modeled with differently colored balls: as the spin-pairs of nucleons must be opposite to each other in the models, chains of protons and neutrons appear within the model (see Figure 13). Also, modelings 


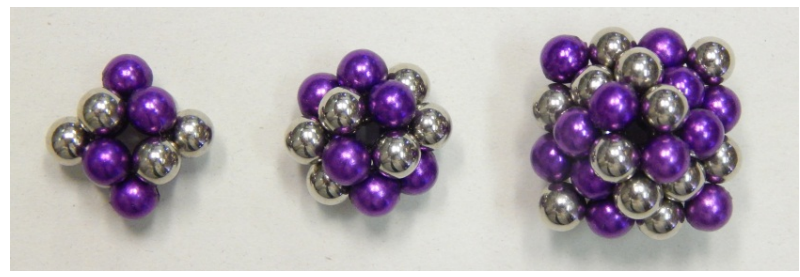

Figure 13. Models of the ${ }^{12} \mathrm{C},{ }^{16} \mathrm{O},{ }^{40} \mathrm{Ca}$ nuclei. The purple/silver colors in this case helps to distinguish the protons/neutrons of the models.

of very light nuclei and heavy nuclei are two fascinating tasks. Thus, there are wide perspectives in the geometric modeling of atomic nuclei.

\section{References}

[1] Conway, J.H. and Sloane, N.J.A. (1993) Sphere Packings, Lattices and Groups. New-York, Springer-Verlag (The Maximum Packing Density Was Hypothesized by Johannes Kepler in 1611 and C.F. Gauss Proved the Lemma for Lattices. While the Perfect Proof for Every Type of Packing Is Still Missing).

[2] Coxeter, H.M.S. (1989) Introduction to Geometry. Wiley.

[3] Sharrad, F., Hamoudi, A. and Radhi, R. (2015) Charge Density Distributions and Form Factors of Some Light Nuclei. Scholar's Press.

[4] Darai, J., Cseh, J. and Jenkins, D.G. (2012) Shape Isomers and Clusterisation in the ${ }^{28}$ Si Nucleus. Physical Review C, 86, Article ID: 064309.

https://doi.org/10.1103/PhysRevC.86.064309

Submit or recommend next manuscript to SCIRP and we will provide best service for you:

Accepting pre-submission inquiries through Email, Facebook, LinkedIn, Twitter, etc. A wide selection of journals (inclusive of 9 subjects, more than 200 journals)

Providing 24-hour high-quality service

User-friendly online submission system

Fair and swift peer-review system

Efficient typesetting and proofreading procedure

Display of the result of downloads and visits, as well as the number of cited articles

Maximum dissemination of your research work

Submit your manuscript at: http://papersubmission.scirp.org/

Or contactwjnst@scirp.org 